\title{
Lavado de activos y su relación con la rentabilidad, banco
}

de la nación, 2020

MBA. Lourdes Guevara Rabanal

lguevarar@ucv.edu.pe

ORCID: 0000-0001-5612-6575

Escuela de Contabilidad

Universidad César Vallejo

Dr. César Augusto Flores Tananta

cflorest@ucvvirtual.edu.pe ORCID: 0000-0002-9336-1483

Escuela de Administración

Universidad César Vallejo

\section{RESUMEN}

Rosero, D. (2015); Indica que el sistema bancario a nivel mundial, tiene la necesidad de incrementar sus medidas de prevención de lavado de activos que permitan formular nuevas acciones. Por eso se conformó en 1989, el Grupo de Acción Financiera Internacional (GAFI). Mientras que en el año 2000 se crea formalmente al Grupo de Acción Financiera de Sudamérica GAFISUD. Por otro lado; El lavado de activos, es un sistema de procesos y supervisiones detallados en la norma, aquellos que se relacionan a la identificación temprana y al reporte de operaciones sospechosas, (Resolución SBS N ${ }^{\circ}$ 2660-2015). Es por ello que se investigó el lavado de activos en entidades financieras y su relación con la rentabilidad; Con la intención de evidenciar las falencias en cuanto al manejo de las operaciones dinerarias y no dinerarias, el objetivo del estudio fue; Determinar la relación entre el lavado de activos y la rentabilidad del banco de la nación; 2020. La metodología que se usó fue: Deducción lógica; técnica, la encuesta; Instrumento, el cuestionario; Diseño del estudio, no experimental de corte transversal, descriptiva correlacional, de tipo aplicada y el resultado más notorio, es que existe una relación positiva (Rho=0.096), entre lavado de activos y la rentabilidad.

Palabras Clave: lavado de activos; rentabilidad; riesgo; gestión y operaciones inusuales 


\title{
Management by results to improve the quality of service in public entities
}

\begin{abstract}
ABSTRAC
Rosero, D. (2015); It indicates that the banking system worldwide has the need to increase its money laundering prevention measures that allow the formulation of new actions. That is why the Financial Action Task Force (FATF) was formed in 1989. While in 2000 the GAFISUD South American Financial Action Group was formally created. Secondly; Money laundering is a system of processes and supervisions detailed in the standard, those that relate to the early identification and reporting of suspicious transactions, (Resolution SBS No. 2660-2015). That is why money laundering in financial entities and its effect on profitability was investigated; With the intention of showing the shortcomings regarding the handling of monetary and non-monetary operations, the objective of the study was; Determine the relationship between money laundering and the profitability of the nation's bank; 2020. The methodology used was: logical deduction; technical, survey; Instrument, the questionnaire; Design of the study, non-experimental, cross-sectional, descriptive, correlational, applied type, The most notable result is that there is a positive relationship ( Rho $=0.096$ ) between money laundering and profitability.
\end{abstract}

Key Words: Money Laundering; Profitability; Risk; Management and Unusual Operations

Artículo recibido: 10 Agosto. 2021 Aceptado para publicación: 07. Setiembre. 2021

Correspondencia: 1guevarar@ucv.edu.pe Conflictos de Interés: Ninguna que declarar 


\section{INTRODUCCIÓN}

La SBS, (2011); Define el lavado de activos como el proceso por el cual es encubierto el origen de los fondos generados de actividades ilícitas (p.6). Cabe precisar que todos los actos de conversión, como la tenencia y traslado de dinero obtenido a partir de actos que importan la comisión de un delito, son punibles y gozan de relevancia jurídico penal (Muñico, Sh; Rebatta, Q; Orihuela, P \& Cornelio A; 2018, p.70). Es por eso que, en los últimos veinticinco años, las instituciones financieras han pasado de un ámbito local o regional a uno de escala transnacional o transfronterizo, en el que las fronteras ya no constituyen obstáculos para sus actividades ilícitas y establecer redes de contacto entre ellas para la colaboración mutua. (Armienta, G, Medina, G y García, 2015, p.29); Sin embargo, Según en un informe emitido por el Ministerio Público durante el año 2017, las fiscalías nacionales especializadas en delitos de lavado de activos recibieron alrededor de 509 denuncias por este ilícito, de los cuales 444 casos fueron resueltos, mientras que 65 aún continúan en trámite (Ministerio público, 2017, p.52);

Actualmente la competitividad de las instituciones es demandante, innovándose constantemente para enfrentar los grandes cambios que forman parte de los requisitos de mantenerse en el mercado de manera eficiente. La implementación de tecnologías, aplicación de políticas socioeconómicas y todo lo que conlleva la palabra globalización, se presta para que existan riesgos en varios campos de las empresas tales como: riesgos financieros, riesgos empresariales o riesgos laborales; creando un ambiente propicio para la proliferación de fraudes en los movimientos económicos (Guerrero, A; Marín, M \& Bonilla, D. 2019)

Por otro lado, la Ley 27765/LA/2002, según DL. № 986/2007 describe en sus artículos 1 y 2 el proceso del Lavado de activos:

Artículo 1: Actos de Conversión y Transferencia, El que convierte o transfiere dinero, bienes, efectos o ganancias cuyo origen ilícito conoce o puede presumir, y dificulta la identificación de su origen, su incautación o decomiso; será reprimido con pena privativa de la libertad no menor de ocho ni mayor de quince años y con ciento veinte a trescientos cincuenta días multa.

Artículo 2: Actos de Ocultamiento y Tenencia, El que adquiere, utiliza, guarda, custodia, recibe, oculta, administra o transporta dentro del territorio de la República o introduce o 
retira del mismo o mantiene en su poder dinero, bienes, efectos o ganancias, cuyo origen ilícito conoce o puede presumir, y dificulta la identificación de su origen, su incautación o decomiso, será reprimido con pena privativa de la libertad no menor de ocho ni mayor de quince años y con ciento veinte a trescientos cincuenta días multa

Es por esto que la SBS (2011); Indica que el lavado de activos y el financiamiento del terrorismo constituyen serios delitos y su efectiva lucha es de prioritario interés del estado peruano, en concordancia con lo señalado por el acuerdo nacional en sus políticas de estado vigésimo sexta y trigésima; Asimismo, El Banco de la Nación, como sujeto obligado de acuerdo con lo establecido en las disposiciones legales vigentes, ha implementado un Sistema de Prevención de Lavado de Activos y del Financiamiento del Terrorismo, el cual cuenta con políticas y procedimientos de control, con el fin de minimizar los riesgos de ser utilizado como intermediario para legitimar fondos provenientes de actividades ilícitas; Asi mismo la GAFISUD 2010, Indica que a partir de lo dispuesto en el apartado II.2 del Capítulo 1-14 RAN SBIF. Que: Es obligación no delegable del banco identificar y conocer a sus clientes. (p.122)

Cabe indicar que, para llevar a cabo esta actividad del lavado de activos, el cliente juega un papel muy importante, el mismo que es definido por Kotler P. (2003) como aquel por quién se planifica, implementa y controlan todas las actividades en las empresas o instituciones financieras (p. 8); Asimismo, la American Marketing Association - A.M.A. (2009), indica que el cliente, es el comprador potencial o real de los productos o servicios que se ofrece en el mercado; Por otro lado, en el Diccionario de Marketing, de Cultural S.A. (1999). encontramos que el cliente, es un Término que define a la persona u organización que realiza una compra para satisfacer sus necesidades; Por otro lado, la GAFI 2012, Define al beneficiario final como la persona natural que finalmente posee o controla a un cliente y/o la persona natural en cuyo nombre se realiza una transacción (p. 119)

Sin embargo, toda entidad financiera necesita de gestión, el mismo que es definido por Black H. (2004), como el proceso de estructurar y utilizar un conjunto de recursos orientados hacia el recurso de metas para llevar a cabo las tareas en un entorno organizacional. De similar forma, lo definen; Robbins \& Coulter (2005), quienes indican que es la coordinación de actividades de trabajo, de modo que se ejecuten de manera eficientemente con otras personas y a través de ellas, Así mismo, Rosario \& Rosario 
(2017), manifiesta que la gestión financiera, es la suministración eficiente de las fuentes o recursos financieros, así como su adecuada planificación con la finalidad de tomar decisiones.

El blanqueamiento del dinero no sería detectable en las gestiones financieras, si es que la gestión administrativa no diera un soporte adecuado, eficaz y oportuno en todas las acciones que el Banco de la Nación realiza, es por eso que, Chiavenato (2006), define a la gestión administrativa, como la ejecución y control de acciones, con el objeto de obtener resultados eficaces y eficientes el mismo que permitan asegurar la utilización óptima de todos los recursos; En efecto, Welsh, Hilton y Gordon (2005), indican que son una serie de acciones autónomas utilizadas por la administración de una organización.

No obstante, todas estas actividades que involucran dinero que provienen de fuentes ilícitas o narcotráfico afectan la rentabilidad del Banco de manera significativa, cuando los clientes son detectados como potenciales blanqueadores de dinero producto de las captaciones, el mismo que involucra al banco y afecta directamente en las colocaciones y por defecto en los intereses diferidos que el banco tenía por cobrar a otros clientes; Según, De la Hoz, Ferrer y de la Hoz (2008), define la rentabilidad como un indicador amplio e importante a nivel internacional, además permite que bajo su análisis financiero se tenga una valuación a una empresa sobre la capacidad que tiene para crear beneficios y así financiar sus operaciones. De este modo tener un diagnóstico sobre la situación de una empresa, permite a los directivos tomar decisiones acertadas, teniendo en cuenta que una decisión inapropiada provocará graves problemas que se verán afectados en la mejora de la economía de la institución.

Al respecto Mafra, Gonzáles, Ricardo, \& Wahrlich (2016), la rentabilidad muestra el desempeño financiero que la empresa cree pertinente para la toma de decisiones sobre financiamiento. Aunado a esto, Hernández \& Ríos (2013), indica que es una variable exógena, es decir una variable específica, ya que afecta la estructura financiera, la combinación de la deuda y el capital destinado al financiamiento, cabe precisar qué para medir el rendimiento de una entidad, se debe controlar costos o gastos y convertir ventas en ganancias, se usan los indicadores de rentabilidad (Herrera, T., De la Hoz, \& Vergara, 2012); De esto se desprende que los índices más utilizados para medir la rentabilidad de acuerdo a Goldman, K. (2017) son: el retorno sobre las ventas (ROS), retorno sobre los 
activos (ROA) y retorno sobre el patrimonio (ROE), para propósito de este artículo se usó el ROA y ROE, por ser más específicos (p. 103-112)

Finalmente, el banco de la nación de la ciudad de Tarapoto, siendo un referente en la banca a nivel del estado, es necesario que se efectué un estudio sobre el nivel de blanqueamiento de dinero que existe en las múltiples operaciones que realizan los clientes y como esto afecta la rentabilidad del banco.

\section{ESTRATEGIAS METODOLÓGICAS O MATERIALES Y MÉTODOS}

La metodología aplicada en este estudio de investigación fue, el lógico deductivo el cual consisten en la utilización de los principios teóricos y normativas vigentes sobre el lavado de activos en el banco de la nación de San Martín, buscando el efecto que tiene en la rentabilidad. La población estuvo conformada por 40 colaboradores y 10 ejecutivos que laboran en la entidad financiera de San Martín, contratados bajo el régimen laboral del D.L 728 - Ley de promoción y competitividad laboral; Por otro lado se analizó todos los datos que recolectamos utilizando la técnica de la encuesta y el instrumento del cuestionario, con la finalidad de conocer y comprender la eficiencia y eficacia de las dimensiones planteadas en la variable de Lavado de activos: Colocación, ensombrecimiento e integración en cuanto a la variable de la calidad de Rentabilidad se consideró las dimensiones de: Rentabilidad sobre el patrimonio - ROE y la rentabilidad sobre la inversión - ROI; Sin embargo, toda investigación científica debe demostrar confiabilidad y seguridad de las instrumentos de aplicación de recolección de datos; Es por eso que, Alarcón R. (1991), define a la confiabilidad y seguridad de una herramienta como el equilibrio y estancia de los resultados esperando que no evidencien cambios significativos en el transcurso de una serie de estudios aplicando el test. Sin embargo, Córdova, I. (2013), manifiesta que un instrumento es confiable y seguro, cuando tiene la habilidad de realizar cálculos de una variable en distintas etapas cercanas. Las técnicas referenciadas más usadas y más confiable por los autores citados con antelación para calcular la seguridad de la herramienta es el Alpha de Cronbach, el mismo que se aplicó como piloto a 10 colaboradores de la entidad financiera; La validez del instrumento se hizo mediante juicio de 3 expertos.

\section{RESULTADOS Y DISCUSIÓN}


Los resultados que se obtuvieron en la presente investigación, se narrarán por dimensiones, tomando en consideración todas las opiniones de los colaboradores y ejecutivos del Banco de la Nación (población encuestada), con la finalidad de ver si se ha logrado el cumplimiento de los objetivos específicos y el objetivo general.

Objetivo específico 1. Analizar el lavado de activos en el Banco de la Nación, 2020.

Tabla 1: Dimensión de colocación de la variable Lavado de activos.

\begin{tabular}{|c|c|c|}
\hline Nivel de Dimensión & Puntaje & Porcentaje \\
\hline Bajo & 6 & $20 \%$ \\
\hline Regular & 8 & $27 \%$ \\
\hline Alto & 16 & $53 \%$ \\
\hline Total & 30 & $100 \%$ \\
\hline
\end{tabular}

Fuente: Elaboración propia

En la tabla $\mathrm{N}^{\circ} 01$, se observa los resultados de la evaluación a 40 colaboradores del banco de la Nación, donde el 20\% manifestaron que la colocación del dinero ilícito es baja, mientras que el $27 \%$ opinó que la colocación del dinero es regular y el $53 \%$ piensa que la colocación del dinero obtenido de fuentes ilícitas, es alta.

Tabla 2: Dimensión de ensombrecimiento de la variable Lavado de activos.

\begin{tabular}{|c|c|c|}
\hline Nivel de Dimensión & Puntaje & Porcentaje \\
\hline Bajo & 6 & $20 \%$ \\
\hline Regular & 20 & $67 \%$ \\
\hline Alto & 4 & $13 \%$ \\
\hline Total & 30 & $100 \%$ \\
\hline
\end{tabular}

Fuente: Elaboración propia

\section{Interpretación}

En la tabla $\mathrm{N}^{\circ} 02$, se observa que los resultados de la evaluación aplicada a 40 colaboradores del banco de la Nación, el 20\% opinaron que el ensombrecimiento del dinero es bajo, mientras que el $67 \%$ consideró que el ensombrecimiento del dinero es regular y el 13\% manifestó que el ensombrecimiento es alta. 
Tabla 3: Dimensión de integración de la variable Lavado de activos.

\begin{tabular}{|c|c|c|}
\hline Nivel de Dimensión & Puntaje & Porcentaje \\
\hline Bajo & 8 & $27 \%$ \\
\hline Regular & 16 & $53 \%$ \\
\hline Alto & 6 & $20 \%$ \\
\hline Total & 30 & $100 \%$ \\
\hline
\end{tabular}

\section{Interpretación}

En la tabla $\mathrm{N}^{\circ} 03$, se observa los resultados de la evaluación a 40 colaboradores del banco de la Nación, donde el $27 \%$ de la población encuestada manifiesta que la integración de activos disponibles y exigibles, es baja, mientras que el 53\% opinó que la integración de los activos disponibles y exigibles, es regular y el $20 \%$ piensa que la integración es alta.

Gráfico 01: Consolidado del Objetivo 1

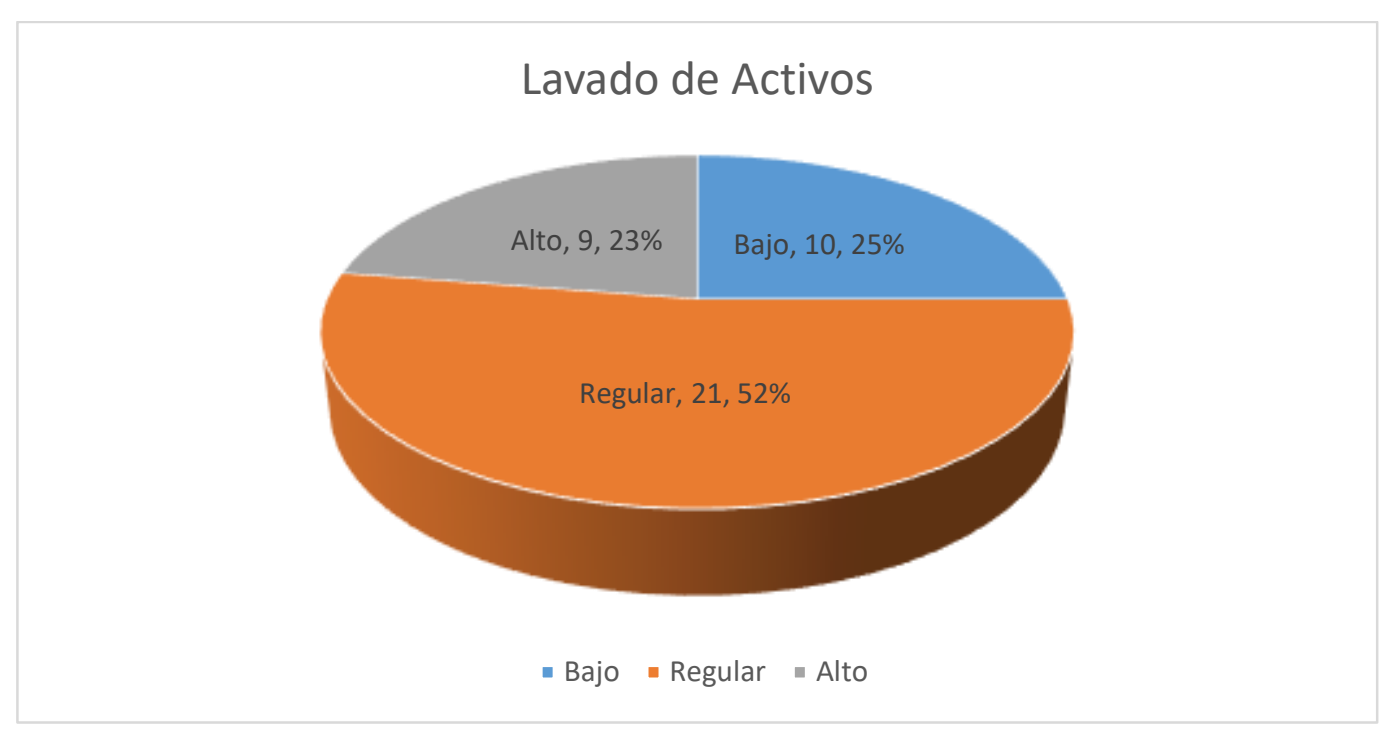

Fuente: Elaboración propia

\section{Interpretación.}

En el gráfico $\mathrm{N}^{\circ} 01$, se observa los resultados de la evaluación a 40 colaboradores del banco de la Nación, donde el 25\% manifestaron que el lavado de activos en la institución es bajo, mientras que el $52 \%$ de los encuestados opinaron que el blanqueamiento del dinero se da manera regular y el $23 \%$ piensa que el lavado de activos es alto.

Cabre precisar que en cuanto a la gestión del lavado de activos, las fases de la colocación, ensombrecimiento e integración del dinero proveniente de fuentes ilícitas por parte de los 
clientes sospechosos de tráfico ilícito de dinero; Sobre el particular, los colaboradores del banco de la nación, indicaron que es alto lo que afecta el nivel de captaciones obtenidos de manera legal y esto afecta la rentabilidad; Tal como afirma, Felaban, (2010-2021), cuando indica que la etapa de la colocación, consiste en la recepción óptica de activos de cualquier naturaleza o de dinero provenientes de actividades ilícitas, asimismo la colocación del dinero dentro del sistema económico y financiero, suele ser el paso más difícil. Los delincuentes reciben grandes cantidades de efectivo de los cuales deben desprenderse rápidamente para evitar la acción de las autoridades; UNODOC (2011), Manifiesta que en la etapa de ensombrecimiento o disposición, buscan que los fondos recaudados queden disponibles para las empresas de acciones terroristas, quedando simplemente a la espera de su utilización final.

Objetivo específico 2: Analizar la rentabilidad en el Banco de la Nación, 2020

Tabla 4: Dimensión de rendimiento sobre la inversión, de la variable de rentabilidad.

\begin{tabular}{|c|c|c|}
\hline Nivel de Dimensión & Puntaje & Porcentaje \\
\hline Bajo & 2 & $20 \%$ \\
\hline Regular & 6 & $60 \%$ \\
\hline Alto & 2 & $20 \%$ \\
\hline Total & 10 & $100 \%$ \\
\hline
\end{tabular}

Fuente: Elaboración propia

\section{Interpretación}

En la tabla $\mathrm{N}^{\circ} 04$, se observa los resultados de la evaluación a 10 ejecutivos del banco de la Nación, donde el 20\% de los encuestados manifestaron que la rentabilidad sobre la inversión es baja, mientras que el $60 \%$ opinó que la rentabilidad sobre la inversión es regular y el $20 \%$ piensa que la rentabilidad es alta.

Tabla 5: Dimensión de rendimiento sobre el patrimonio de la variable rentabilidad

\begin{tabular}{lcc}
\hline Nivel de Dimensión & Puntaje & Porcentaje \\
Bajo & 1 & $10 \%$
\end{tabular}




\begin{tabular}{llll}
$\begin{array}{l}\text { Regular } \\
\text { Alto }\end{array}$ & 5 & $50 \%$ \\
& & 4 & $40 \%$ \\
\hline & Total & 10 & $100 \%$
\end{tabular}

\section{Fuente: Elaboración propia}

\section{Interpretación}

En la tabla $\mathrm{N}^{\circ} 5$, se observa los resultados de la evaluación a 10 ejecutivos del banco de la Nación, donde el 10\% manifestaron que la rentabilidad sobre el patrimonio es baja, mientras que el $50 \%$ opinó que la rentabilidad sobre el patrimonio es regular y el $40 \%$ piensa que rentabilidad sobre el patrimonio es alta

\section{Gráfico $\mathbf{N}^{\circ}$ 2: Consolidado del objetivo 2}

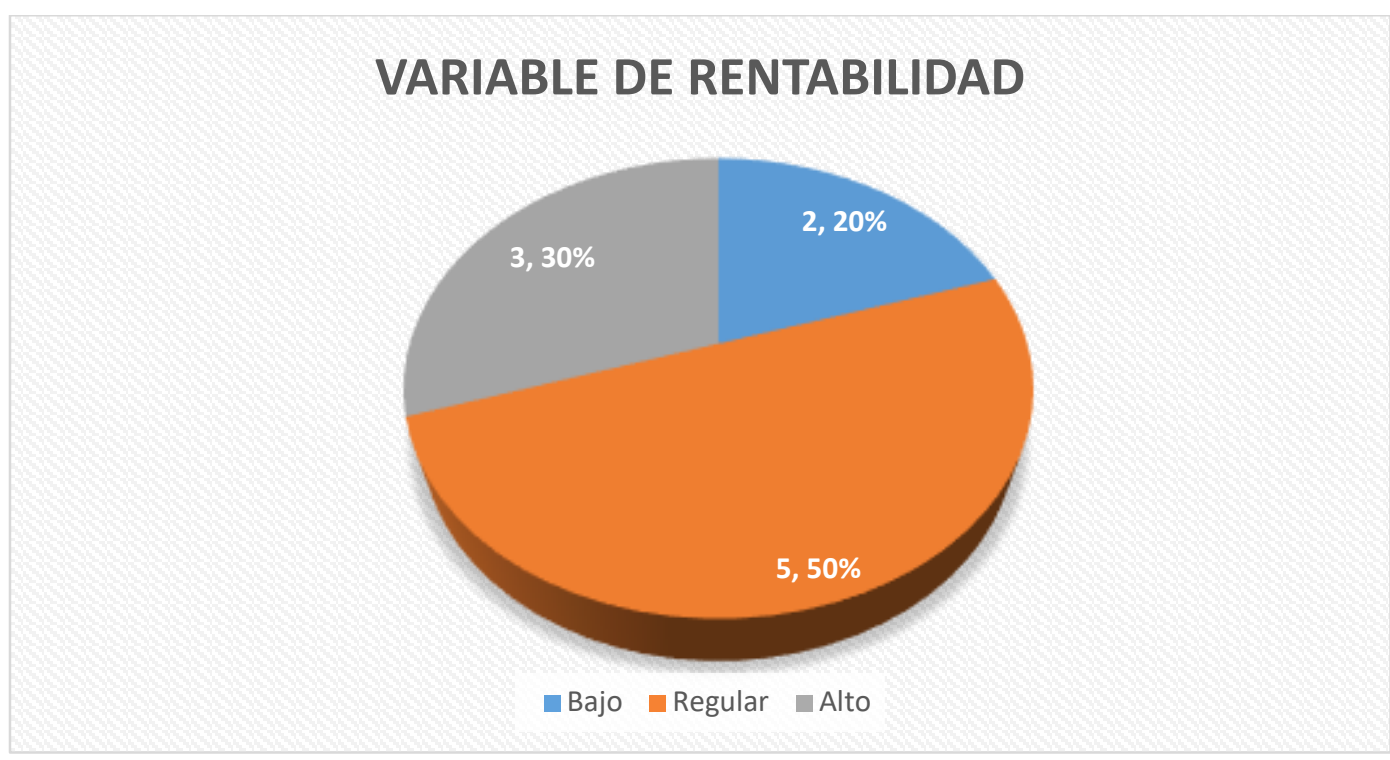

Fuente: Elaboración propia

Tal como se observa en gráfico $\mathrm{N}^{\circ} 02$, los resultados de la evaluación a 10 ejecutivos del banco de la nación, se observa que $20 \%$ consideró que la rentabilidad en el banco de la nación es baja, mientras que el 30\% indicó que la rentabilidad es regular y el 50\% opinó que la rentabilidad del banco es alta.

La rentabilidad es el resultado positivo, producto de la aplicación de estrategias que obtiene la empresa luego de haber identificado sus costes y gastos, utilizando las diferentes acciones destinado a la producción e inversión con todos los ingresos adquiridos en el periodo (Gitman \& Zutter, 2012). Sin embargo, los ejecutivos del banco, el $50 \%$ manifestaron que la rentabilidad es regular y una empresa que se encuentre en 
condiciones financieras regulares, es preocupante tal como lo manifiesta; Jara, Sánchez, Bucaram, \& García, (2018), en su investigación, cuando define a la rentabilidad como la predisposición que tienen las entidades para generar ganancias interrelacionadas con diversos factores que usan en sus operaciones

Objetivo específico 03: Identificar las dimensiones de la variable del lavado de activos que tiene menor relación con la rentabilidad del Banco de la Nación, 2020.

Tabla $\mathbf{N}^{\circ}$ 06: Medición de las dimensiones de integración y rendimiento sobre la inversión

\begin{tabular}{ccc}
\hline $\begin{array}{c}\text { Dimensiones de } \\
\text { Integración }\end{array}$ & $\begin{array}{c}\text { Relación con Rentabilidad } \\
\text { (Rho de Spearman) }\end{array}$ & Rango de la relación \\
\hline ROI &,- 016 & Correlación negativa muy baja \\
Integración &, 064 & Correlación positiva muy baja \\
\hline
\end{tabular}

Fuente: Archivo recuperado, cuestionario procesado en el Software IBM SPSS Statistics 26.

\section{Interpretación}

De acuerdo a la Tabla $\mathrm{N}^{\circ}$ 06, las dimensiones de la integración o disposición del dinero que tienen menor relación con la rentabilidad en el banco de la nación, de acuerdo a los resultados mostrados en la tabla; es la rentabilidad sobre la inversión, con un Rho Spearman de -0,016 mostrando una correlación negativa nivel medio y la integración con un Rho Spearman de 0,064 mostrando una correlación positiva considerable; Tal como lo manifiesta, Anderson (1999), donde indica que el coeficiente de correlación de parámetros de Spearman puede ubicarse desde -1.0 hasta +1.0 , y se interpreta así: los valores más próximos a +1.0 , el mismo que considera que existe una sólida asociación entre los resultados, o sea que en la medida que aumenta un rango el otro también aumenta; en cambio los valores próximos a -1.0 señalan que hay una enérgica asociación negativa entre los resultados, vale decir que al incrementar un rango, el otro disminuye. cuando el valor es 0.0 , no existe correlación.

Objetivo General. Determinar la relación entre el lavado de activos y la rentabilidad del banco de la nación; 2020.

Tabla 7: Correlación entre el lavado de activos y la rentabilidad. 


\begin{tabular}{cllcc}
\hline & & Coeficiente de & 1,000 &, 096 \\
& Lavado de & correlación & & \\
Rho de & Activos & Sig. (bilateral) &. &, 932 \\
Spearman & & N Encuestados $^{\circ}$ & 40 & 10 \\
& & Coeficiente de &, 096 & 1,000 \\
& Rentabilidad & correlación & & \\
& & Sig. (bilateral) &, 932 & 10 \\
\hline
\end{tabular}

Fuente: Archivo recuperado, del cuestionario procesado en el Software IBM SPSS

Statistics26.

\section{Interpretación}

En la descripción de la tabla número 7, se puede observar que la significancia bilateral de los datos fue menor que el nivel " $p$ " valor propuesto $(\mathrm{p}=0.932<0.05)$, este efecto permite tomar la decisión de rechazar la Ho y aceptar la Hi, Además, el Rho Spearman obtuvo un valor de $(\mathrm{Rho}=0,096)$ mostrando una correlación positiva perfecta, es decir cuando los clientes blanquean demasiado dinero, la rentabilidad disminuye, porque esto afecta la correcta medición del dinero lícito de fuentes confiables, el mismo que el banco no podría colocar en los clientes a través de los diferentes servicios que prestan y por ende afecta la captación de ingresos que generan los intereses. Asimismo, Hernández \& Fernández (1998), Indican que es ineludible tener en circunspección la importancia del valor de "rs", dada por el valor de "p" que lo acompaña. Cuando el valor de "p" es menor que 0.05, se puede concluir que la correlación es significativa, lo que indica una relación real, no debida al azar.

\section{CONCLUSIÓN O CONSIDERACIONES FINALES}

La investigación después del proceso de revisión de diferentes fuentes y aplicación de instrumentos a los colaboradores y ejecutivos, se indica que existe una correlación positiva perfecta $(\mathrm{Rho}=0.096)$, entre el lavado de activos y la rentabilidad del banco de la nación, 2020.

El lavado de activos en cuanto a las diferentes etapas que atraviesa, en cuanto a la colocación es alto con tendencia a regular, en cuanto a la fase del ensombrecimiento, es regular con tendencia baja y en cuanto a la integración es regular con tendencia baja, lo que significa que hay algunos aspectos por mejorar en procesos de control e identificación de posibles clientes o empresas relacionadas con el narcotráfico. 
La rentabilidad sobre la inversión en la institución financiera, es regular con tendencia al alza, de similar forma la contabilidad sobre el patrimonio, se encuentra en condiciones regulares con tendencia al alza.

La relación que existe entre las dimensiones de la variable de integración que tenga menor relación con la rentabilidad, es baja y esto se debe a que el personal es capacitado y se cumplen las políticas del banco.

\section{LISTA DE REFERENCIAS}

Anderson, D. R., Sweeney, D. J., \& Williams, T. A. (1999). Estadística para administración y economía. México: International Thomson Editores

Armienta, G., Goite, M., Medina, A., Gambino, L., \& García, L. (2015). El lavado de dinero en el siglo XXI una visión desde los instrumentos jurídicos internacionales, la doctrina y las leyes en América Latina y España. México: Universidad Autónoma de Sinaloa

Chiavenato, I. (2004), En su libro Introducción a la teoría general de la administración, 7ma. Ed. editorial Mc-Graw Hill Interamericana, Mexico.

Daza, J. (2015). Análisis de la interrelación crecimiento-rentabilidad en Brasil. Tourism \& Management Studies, 11(2), 182-188. doi: DOI: 10.18089/tms.2015.11222

Diaz, M. (2010). El Análisis de los estados contables en un entorno dinámico y gerencial de la empresa. Universo Contabilidad, 62, 121-140

Del sitio web "Marketingpower.com", de la American Marketing Association (A.M.A.), Sección: Resource Library - Dictionary.

Recuperado: http://www.marketingpower.com/_layouts/Dictionary.aspx?dLetter=C, obtenido en fecha: 22 de julio 2009.

Del «Diccionario de Marketing», de Cultural S.A., Edición 1999, Pág. 54.

De La Hoz, B., Ferrer, M., \& De La Hoz, A. (2008). Indicadores de rentabilidad: herramientas para la toma de decisiones financieras en hoteles de categoria media ubicados en Maracaibo. Revista de Ciencias Sociales, XIV(1), 88-109. Retrieved from https://www.redalyc.org/pdf/280/280116 73008.pdf

Federación latinoamericana de bancos FELABAN (2015), Sistemas de Prevención de Lavado de Activos. Recuperado: http://www.felaban.com/lvdo/cap1.html

Gitman, L., \& Zutter, C. (2012). Principios de la Administración financiera (doceava ed.). 
México: Pearson Educación.

García, D. (2014). Ratios y márgenes de rentabilidad. Un caso práctico en el sector de la construcción. Revista Contable $(18,56)$.

García Cárdenas, S. C. (2018). El delito de peculado y su implicancia en el delito de lavado de activos en el sistema penal peruano.

González Martín, D., \& González García, M. C. (2016). Los paraísos fiscales.

Guerrero, A., Marín, M. y Bonilla, D. (2019), The laundering of assets and their influence on the productivity and economic development of a country, Articulo científico, Vol. N 40, año 2018.

GAFISUD. (2006), En el Informe de evaluación mutua sobre lavado de activos y financiamiento del terrorismo. Chile. Recuperado: http://www.gafisud. info/documentos/esp/evaluaciones_mutuas/Chile_2nda_Ronda_2006.pdf [visitado el 11/3/2016.

GAFI (2007) p. 4; Rodríguez y Germán (2001) p. 35; y Lombardero (2009) p. 112.

GAFISUD (2010) Indica que a partir de lo dispuesto en el apartado II.2 del Capítulo 114 RAN SBIF que indica que: "Es obligación no delegable del banco identificar y conocer a sus clientes. p. 122.

GAFI (2012) p. 119, El beneficiario final es "las personas naturales que finalmente posee o controla a un cliente y/o la persona natural en cuyo nombre se realiza una transacción.

Gerencia de Oficialía de Cumplimiento del Banco de la Nación (2016) Manual de Prevención y Gestión de los Riesgos de Lavado de Activos y del Financiamiento del Terrorismo del Banco de la Nación, Perú.

Goldman, K. (2017). Financial liquidity and profitability management in practice of Polish Business. Financial Environment and Business Development, 4, 103-112. https://doi.org/https://doi.org/10.1007/97 8-3-319-39919-5_9

Gerencia de Oficialía de Cumplimiento del Banco de la Nación (2016) Manual de Prevención y Gestión de los Riesgos de Lavado de Activos y del Financiamiento del Terrorismo del Banco de la Nación, Perú.

Huacchillo Pardo, L. A., Ramos Farroñan, E. V., \& Pulache Lozada, J. L. (2020). La gestión financiera y su incidencia en la toma de decisiones financieras. Universidad y Sociedad, 12(2), 356-362 
Hernández, G., \& Ríos, H. (2013). Estructura financiera óptima, en la industria de los alimentos, que cotiza en la Bolsa Mexicana de Valores. Econoquantum, 10(2), 77-97. https://doi.org/10.18381/eq.v10i2.163

Herrera, T., De la Hoz, E., \& Vergara, J. C. (2012). Aplicación de análisis discriminante para evaluar el mejoramiento de los indicadores financieros en las empresas del sector alimento de Barranquilla-Colombia. Revista Chilena de Ingeniería, 20(3), 320-330. Retrieved from http://search.ebscohost.com/login.aspx? direct $=$ true $\& d b=$ fua $\& A N=85224980 \&$ site $=$ ehost-live

Hernández, R., Fernández, C., \& Baptista, M. (2010). Metodología de la investigación (Quinta edición ed.). México D.F.: McGRAW-HILL.

Hernández, R., Fernández, C., \& Baptista, M. (2010). METODOLOGÍA de la investigación (Quinta edición ed.). México D.F.: McGRAW-HILL

Hernández Sampieri, R., \& Fernández Collado, C. (1998). Metodología de la investigación. México: McGraw-Hill.

Hit, M., Black, S. y Porter L. (2006), en su libro de Administración, 9na. Ed. Editorial Pearson educación. Mexico.

Kotler P. (2003), en su libro Los 80 Conceptos Esenciales del Marketing de la A a la Z, , Pearson Educación S.A., Págs. 8 y 9

Muñico, Sh., Rebatta, Q., Orihuela, P. y Cornelio, O. (2018), En su investigación la Lucha contra el lavado de activos ¿Estamos avanzando o retrocediendo? ¿Realmente nuestras autoridades están luchando contra la criminalidad? ¿Qué falta? Recuperado: : http://dx.doi.org/10.18259/iet.2018005

Ministerio Público. (2017). Anuario estadístico. Lima: Orace.

Ministerio Público. (2013). Anuario estadístico. Lima: Orace

Mafra, V., Gónzales, E., Ricardo, P., \& Wahrlich, R. (2016). A cost-benefit analysis of three gillnet fisheries in Santa Catarina, Brazil: contributing to fisheries management decisions. Latin American Journal of Aquatic Research, 44(5), 1096-1115. https://doi.org/10.3856/vol44-issue5fulltext-19

Pontón, C. (2013). La economía del narcotráfico y su dinámica en América Latina. Iconos. Revista de Ciencias Sociales

Rosero, D. (2015), en su tesis par aobtener el grado de maestría Modelo de gestión y 
lavado de activos en instituciones financieras, Universidad Técnica de Ambato Ecuador.

Recuperado: http://repositorio.uta.edu.ec/jspui/handle/123456789/20469

Resolución SBS Nº 2660 (2015), Que, mediante la Ley N²7693, se crea la Unidad de Inteligencia Financiera del Perú, UIF-Perú, encargada de recibir, analizar, tratar, evaluar y transmitir información para la detección del lavado de activos y/o del financiamiento del terrorismo (LA/FT).

Reglamento de Gestión de Riesgos de Lavado de Activos y del Financiamiento del Terrorismo, aprobado por resolución de la Superintendencia de Banca, Seguros y AFP de la República del Perú (SBS) No 2660-2015. Diario Oficial El Peruano, 18 de mayo de 2015.

Resolución exenta de la Unidad de Análisis Financiero, DJ Nº 107-038-2013, de 31 de enero de 2013.

Resolución exenta de la Unidad de Análisis Financiero DJ Nº 107-597-2013, de 26 de agosto de 2013

Robbins, S. y Coulter, M. (2005) en su libro de Administración, 8va. Ed. Editorial Pearson educación. Mexico.

Rosario, H., \& Rosario, F. (2017). Gestión financiera. Editorial Universidad de Almería.

Superintendencia de Banca y Seguros - SBS. (2011), Plan nacional de lucha contra el lavado de activos y el financiamiento del terrorismo.

Recuperado: https://www.peru.gob.pe/normas/docs/Plan\%20Nacional.pdf

Superintendencia de banca y seguros (2011), Plan Nacional de Lucha contra el Lavado de Activos y el financiamiento del terrorismo p.2

Tordoya Romero, H. (2015). Inteligencia financiera y el lavado de activos en el Perú.

Urrutia, A., Heriberto, J., Arévalo A, \& Gamero Figueroa, M. A. (2018). La evasión fiscal como indicio del cambio de calificación jurídica del delito de lavado de dinero y activos (Doctoral dissertation, Universidad de El Salvador).

Welsh, Hilton y Gordon (2005), en su libro de Presupuesto, Planificación y control Sexta ed. Editorial Pearson educación. Mexico. 